\title{
Certain integrals involving extended Bessel-Maitland function associated with Jacobi polynomials
}

\author{
Musharraf Ali ${ }^{1}$ and Waseem A. Khan ${ }^{2}$ \\ ${ }^{1}$ Department of Mathematics, G. F. College, Shahjahanpur, India \\ ${ }^{2}$ Department of Mathematics and Natural Sciences, Prince Mohammad Bin Fahd University, Al Khobar, Kingdom of Saudi Arabia
}

Received: 22 June 2019, Accepted: 25 October 2019

Published online: 18 February 2020.

\begin{abstract}
The intent of the paper is to establish some interesting integrals involving the product of generalized Bessel-Maitland function with Jacobi polynomial, which are expressed in terms of generalized hypergeometric function. Some special cases are deduced.
\end{abstract}

Keywords: Jacobi polynomial, generalized hypergeometric function, Wright generalized Bessel-Maitland function.

\section{Introduction}

In the last decade, many authors (see, e.g., [1-19] have developed numerous integral formulas involving a variety of special functions. Also many integral formulas associated with the Bessel functions of several kinds have been presented (see, e.g., [1-7]). Those integrals involving Bessel-Maitland functions are not only of great interest to the pure mathematics, but they are often of extreme importance in many branches of theoretical and applied physics and engineering (see [12]). Several methods for evaluating infinite or finite integrals involving Bessel-Maitland functions have been known (see, e.g.,[1] and [17]). However, these methods usually work on a case-by-case basis.

Currently, Ghayasuddin and Khan [1], Khan et al. [2-4, 7], Ali et al. [4-6] gave certain interesting new class of integral formulas involving the generalized Bessel-Maitland function, which are expressed in terms of the generalized (Wright) hypergeometric function. In the present sequel to the aforementioned investigations, we present two generalized integral formulas involving generalized Bessel-Maitland functions, which are expressed in terms of the generalized (Wright) hypergeometric function. Some special cases and the (potential) usefulness of our main results are also considered and remarked, respectively.

The Bessel-Maitland function $J_{v}^{\mu}(z)$ [18;Eq.(8.3)] defined by the following series representation:

$$
J_{v}^{\mu}(z)=\sum_{n=0}^{\infty} \frac{(-z)^{n}}{n ! \Gamma(\mu n+v+1)}=\phi(\mu, v+1 ;-z)
$$

Singh et al. [17] introduced the following generalization of Bessel-Maitland function as:

$$
J_{v, q}^{\mu, \gamma}=\sum_{n=0}^{\infty} \frac{(\gamma)_{q n}(-z)^{n}}{\Gamma(\mu n+v+1) n !}
$$


where $\mu, v, \gamma \in \mathbb{C}, \operatorname{Re}(\mu) \geq 0, \operatorname{Re}(v) \geq-1, \operatorname{Re}(\gamma) \geq 0$ and $q \in(0,1) \cup \mathbb{N}$ and $(\gamma)_{0}=1,(\gamma)_{q n}=\frac{\Gamma(\gamma+q n)}{\Gamma(\gamma)}$ denotes the generalized Pochhammer symbol.

Recently, Ghayasuddin and Khan [1] introduced and investigated generalized Bessel-Maitland function defined as

$$
J_{v, \gamma, \delta}^{\mu, q, p}(z)=\sum_{n=0}^{\infty} \frac{(\gamma)_{q n}(-z)^{n}}{\Gamma(\mu n+v+1)(\delta)_{p n}},
$$

where $\mu, v, \gamma, \delta \in \mathbb{C}, \Re(\mu) \geq 0, \Re(v) \geq-1, \Re(\gamma) \geq 0, \Re(\delta) \geq 0 ; p, q>0$ and $q<\Re(\alpha)+p$.

In particular Khan et al. [7] introduced and investigated a new extension of Bessel-Maitland function as follows:

$$
J_{\alpha, \beta, v, \sigma, \delta, p}^{\mu, \rho, \gamma, q}(z)=\sum_{n=0}^{\infty} \frac{(\mu)_{\rho n}(\gamma)_{q n}(-z)^{n}}{\Gamma(n \beta+\alpha+1)(\delta)_{p n}(v)_{n \sigma}}
$$

where $\alpha, \beta, \mu, \rho, v, \gamma, \sigma, \delta \in \mathbb{C} ; \Re(\alpha)>0, \Re(\beta)>0, \Re(\rho)>0, \Re(\mu)>0, \Re(v)>0, \Re(\alpha) \geq-1, \Re(\gamma)>0$, $\mathfrak{R}(\delta)>0, \mathfrak{R}(\sigma)>0 ; p, q>0$, and $q<\mathfrak{R}(\alpha)+p$.

\subsection{Relation with Mittag-Leffler functions}

(i) On replacing $\alpha$ by $\alpha-1$ in (1.4), we get the following interesting relation:

$$
J_{\alpha-1, \beta, v, \sigma, \delta, p}^{\mu, \rho, \gamma, q}(-z)=E_{\alpha, \beta, v, \sigma, \delta, p}^{\mu, \rho, \gamma, q}(z)
$$

where $E_{\alpha, \beta, v, \sigma, \delta, p}^{\mu, \rho, \gamma, q}(z)$ is the Mittag-Leffler function defined by Khan and Ahmed [8].

(ii) On setting $\mu=v=\sigma=\rho=1$ and replacing $\alpha$ by $\alpha-1$ in 4 , we get

$$
J_{\alpha-1, \beta, 1,1, \delta, p}^{1,1, \gamma, q}(-z)=E_{\alpha, \beta, p}^{\gamma, \delta, q}(z)
$$

where $E_{\alpha, \beta, p}^{\gamma, \delta}(z)$ is the Mittag-Leffler function defined by Salim and Faraz [15].

(iii) On setting $\mu=v=\sigma=\rho=\delta=p=1$ and replacing $\alpha$ by $\alpha-1$ in 4 , we get

$$
J_{\alpha-1, \beta, 1,1,1,1}^{1,1, \gamma, q}(-z)=E_{\alpha, \beta}^{\gamma, q}(z)
$$

where $E_{\alpha, \beta}^{\gamma, q}(z)$ is the Mittag-Leffler function defined by Shukla and Prajapati [13].

(iv) On setting $\mu=v=\sigma=\rho=\delta=p=q=1$ and replacing $\alpha$ by $\alpha-1$ in 4 , we get

$$
J_{\alpha-1, \beta, 1,1,1,1}^{1,1, \gamma, 1}(-z)=E_{\alpha, \beta}^{\gamma}(z)
$$

where $E_{\alpha, \beta}^{\gamma}(z)$ is the Mittag-Leffler function defined by Prabhakar [10].

(v) On setting $\mu=v=\sigma=\rho=\delta=\gamma=p=q=1$ and replacing $\alpha$ by $\alpha-1$ in 4 , we get

$$
J_{\alpha-1, \beta, 1,1,1,1}^{1,1,1,1}(-z)=E_{\alpha, \beta}(z)
$$

where $E_{\alpha, \beta}(z)$ is the Mittag-Leffler function defined by Wiman [19]. 
(vi) On setting $\mu=v=\sigma=\rho=\delta=\gamma=p=q=1, \alpha=0$ and replacing $\alpha$ by $\alpha-1$ in 4 , we get

$$
J_{0, \beta, 1,1,1,1}^{1,1,1,1}(-z)=E_{\beta}(z)
$$

where $E_{\beta}(z)$ is the Mittag-Leffler function defined by Ghosta Mittag-Leffler [9].

\section{Integrals involving exteded Bessel-Maitland with Jacobi polynomials}

The Jacobi polynomial $P_{n}^{(\alpha, \beta)}(z)$ is defined by (see [11], [14]):

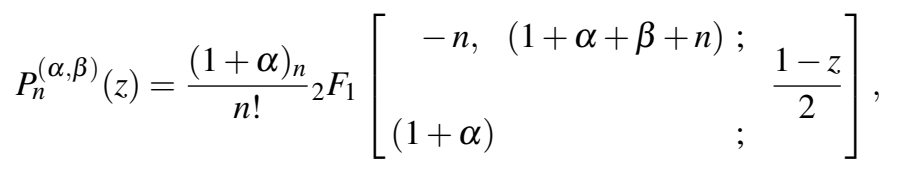

or equivalently

$$
P_{n}^{(\alpha, \beta)}(z)=\sum_{k=0}^{n} \frac{(1+\alpha)_{n}(1+\alpha+\beta)_{n+k}}{k !(n-k) !(1+\alpha)_{k}(1+\alpha+\beta)_{n}}\left(\frac{z-1}{z}\right)^{k} .
$$

From 11 and 12, we find

$$
P_{n}^{(\alpha, \beta)}(1)=\frac{(1+\alpha)_{n}}{n !},
$$

where $P_{n}^{(\alpha, \beta)}(z)$ is a polynomial of degree precisely $n$.

In this section, we define some interesting integral formulas involving a product of extension Bessel-Maitland function and Jacobi polynomials as follows

$$
\begin{aligned}
I_{1} & =\int_{-1}^{1} x^{\lambda}(1-x)^{\alpha}(1+x)^{\delta} P_{n}^{(\alpha, \beta)}(x) J_{\eta, \varepsilon, v, \sigma, \omega, p}^{\mu, \rho, \gamma, q}\left[z(1+x)^{h}\right] d x \\
& =\int_{-1}^{1} x^{\lambda}(1-x)^{\alpha}(1+x)^{\delta} P_{n}^{(\alpha, \beta)}(x) \sum_{k=0}^{\infty} \frac{(\gamma)_{q k}(-z)^{k}(\mu)_{\rho k}(1+x)^{h k}}{\sqrt{(\eta k+\varepsilon+1)(\omega)_{p k}}(v)_{\sigma k}} d x .
\end{aligned}
$$

Interchanging the order of summation and integration, we can write above expression

$$
\sum_{k=0}^{\infty} \frac{(\mu)_{\rho k}(\gamma)_{q k}(-z)^{k}}{\Gamma(\eta k+\varepsilon+1)(v)_{\sigma k}(\omega)_{p k}} \int_{-1}^{1} x^{\lambda}(1-x)^{\alpha}(1+x)^{\delta+k h} P_{n}^{(\alpha, \beta)}(x) d x
$$

Using the formula given in ([12], p.52):

$$
\begin{aligned}
& \int_{-1}^{1} x^{\lambda}(1-x)^{\alpha}(1+x)^{\delta} P_{n}^{(\alpha, \beta)}(x) d x=\frac{(-1)^{n} 2^{\alpha+\delta+1} \Gamma(\delta+1) \Gamma(\alpha+n+1) \Gamma(\delta+\beta+1)}{n ! \Gamma(\delta+\beta+n+1) \Gamma(\delta+\alpha+n+2)} \\
& \times{ }_{3} F_{2}\left[\begin{array}{crr}
-\lambda, & \delta+\beta+1, & \delta+1 ; \\
\delta+\beta+n+1, \delta+\alpha+n+2 ; & 1
\end{array}\right] .
\end{aligned}
$$


From 15 and 16, we find

$$
\begin{aligned}
& I_{1}=\frac{(-1)^{n} 2^{\alpha+\delta+1} \Gamma(\delta+k h+1) \Gamma(\alpha+n+1) \Gamma(\delta+k h+\beta+1)}{n ! \Gamma(\delta+k h+\beta+n+1) \Gamma(\delta+k h+\alpha+n+2)} J_{\eta, \varepsilon, v, \sigma, \omega, p}^{\mu, \rho, \gamma, q}\left[z(2)^{h}\right]
\end{aligned}
$$

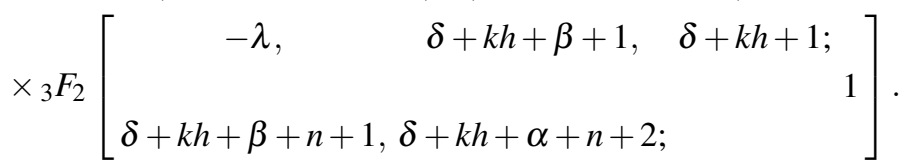

Provided

(i) $\eta, \varepsilon, v, \sigma, \omega, \mu, \rho, \gamma \in \mathbb{C}$;

$\Re(\mu) \geq 0, \Re(\varepsilon) \geq-1, \mathfrak{R}(\gamma) \geq 0, \mathfrak{R}(\rho) \geq 0, \Re(\eta) \geq 0, \Re(\sigma) \geq 0, \Re(\omega) \geq 0, \Re(v) \geq 0 ; p, q>0$ and $q<\Re(\alpha)+1$.

(ii) $\mathfrak{R}(\alpha)>-1$ and $\mathfrak{R}(\beta)>-1$.

$$
\begin{aligned}
I_{2} & =\int_{-1}^{1}(1-x)^{\delta}(1+x)^{\beta} P_{n}^{(\alpha, \beta)}(x) P_{m}^{(\rho, \sigma)}(x) J_{\eta, \lambda, v, \varepsilon, \omega, p}^{\mu, \xi, \gamma, q}\left[z(1-x)^{h}\right] d x \\
& =\sum_{k=0}^{\infty} \frac{(\mu)_{\xi k}(\gamma)_{q k}(-z)^{k}}{\Gamma(\eta k+\lambda+1)(v)_{\varepsilon k}(\omega)_{p k}} \int_{-1}^{1}(1-x)^{\delta+k h}(1+x)^{\beta} P_{n}^{(\alpha, \beta)}(x) P_{m}^{(\rho, \sigma)}(x) d x .
\end{aligned}
$$

Using 12 in above expression, we get

$$
\sum_{k=0}^{\infty} \frac{(\mu)_{\xi_{k}}(\gamma)_{q k}(-z)^{k}}{\Gamma(\eta k+\lambda+1)(v)_{\varepsilon k}(\omega)_{p k}} \frac{(1+\rho)_{m}}{m !} \sum_{k=0}^{\infty} \frac{(-m)_{k}(1+\rho+\sigma+m)_{k}}{(1+\rho)_{k} 2^{k} k !} \times \int_{-1}^{1}(1-x)^{\delta+k h+k}(1+x)^{\beta} P_{n}^{(\alpha, \beta)}(x) d x
$$

Again using 12 in 20, we get

$$
\begin{aligned}
\sum_{k=0}^{\infty} & \frac{(\mu)_{k k}(\gamma)_{q k}(-z)^{k}}{\Gamma(\eta k+\lambda+1)(v)_{\varepsilon k}(\omega)_{p k}} \frac{\Gamma(1+\rho+m) \Gamma(1+\alpha+n)}{m ! n !} \\
& \times \sum_{k=0}^{\infty} \frac{(-m)_{k}(-n)_{k}(1+\rho+\sigma+m)_{k}(1+\alpha+\beta+n)_{k}}{\Gamma(1+\rho+k) \Gamma(1+\alpha+k) 2^{2 k}(k !)^{2}} \times \int_{-1}^{1}(1-x)^{\delta+k h+2 k}(1+x)^{\beta} d x .
\end{aligned}
$$

Using the formula

$$
\int_{-1}^{1}(1-x)^{n+\alpha}(1+x)^{n+\beta} d x=2^{2 n+\alpha+\beta+1} B(1+\alpha+n, 1+\beta+n) .
$$

Here 22 becomes,

$$
\begin{gathered}
I_{2} \frac{2^{\delta+\beta+1} \Gamma(1+\rho+m) \Gamma(1+\alpha+n)}{m ! n !} \sum_{k=0}^{\infty} \frac{(-m)_{k}(-n)_{k}(1+\rho+\sigma+m)_{k}(1+\alpha+\beta+n)_{k}}{\Gamma(1+\rho+k) \Gamma(1+\alpha+k)(k !)^{2}} \\
\times J_{\eta, \lambda, v, \varepsilon, \omega, p}^{\mu, \xi, \gamma, q}\left[z(2)^{h}\right] B(1+\delta+k h+2 k, 1+\beta) .
\end{gathered}
$$

Provided

(i) $\xi, \eta, \varepsilon, v, \sigma, \omega, \mu, \rho, \gamma, \lambda \in \mathbb{C}$;

$\mathfrak{R}(\mu) \geq 0, \mathfrak{R}(\lambda) \geq-1, \mathfrak{R}(\gamma) \geq 0, \mathfrak{R}(\rho) \geq 0, \mathfrak{R}(\eta) \geq 0, \mathfrak{R}(\sigma) \geq 0, \mathfrak{R}(\omega) \geq 0, \mathfrak{R}(v) \geq 0 ; p, q>0$ and $q<\mathfrak{R}(\alpha)+1$. 
(ii) $) \mathfrak{R}(\alpha)>-1$ and $\mathfrak{R}(\beta)>-1$.

$$
\begin{aligned}
I_{3}= & \int_{-1}^{1}(1-x)^{\rho}(1+x)^{\sigma} P_{n}^{(\alpha, \beta)}(x) J_{\eta, \lambda, v, \varepsilon, \omega, p}^{\mu, \xi, \gamma, q}\left[z(1-x)^{h}(1+x)^{t}\right] d x \\
= & \int_{-1}^{1}(1-x)^{\rho}(1+x)^{\sigma} P_{n}^{(\alpha, \beta)}(x) \sum_{k=0}^{\infty} \frac{(\mu)_{\xi k}(\gamma)_{q k}(-z)^{k}}{\Gamma(\eta k+\lambda+1)(v)_{\varepsilon k}(\omega)_{p k}}(1-x)^{k h}(1+x)^{t k} d x . \\
& \sum_{k=0}^{\infty} \frac{(\mu)_{\xi k}(\gamma)_{q k}(-z)^{k}}{\Gamma(\eta k+\lambda+1)(v)_{\varepsilon k}(\omega)_{p k}} \int_{-1}^{1}(1-x)^{\rho+h k}(1+x)^{\sigma+t k} P_{n}^{(\alpha, \beta)}(x) d x .
\end{aligned}
$$

Now, by using 12 in 24 , we get

$$
\sum_{k=0}^{\infty} \frac{(\mu)_{\xi k}(\gamma)_{q k}(-z)^{k}}{\Gamma(\eta k+\lambda+1)(v)_{\varepsilon k}(\omega)_{p k}} \frac{(1+\alpha)_{n}}{n !} \sum_{k=0}^{\infty} \frac{(-n)_{k}(1+\alpha+\beta+n)_{k}}{(1+\alpha)_{k} 2^{k} k !} \times \int_{-1}^{1}(1-x)^{\rho+k h+k}(1+x)^{\sigma+k t} d x .
$$

Using 22 in 25 , we get

$$
I_{3}=\frac{2^{\rho+\sigma+1}(1+\alpha)_{n}}{n !} \sum_{k=0}^{\infty} \frac{(-n)_{k}(1+\alpha+\beta+n)_{k}}{(1+\alpha)_{k}(k !)} \times J_{\eta, \lambda, v, \varepsilon, \boldsymbol{\xi}, \boldsymbol{\gamma}, p}^{,, q}\left[z(2)^{h+t}\right] B(1+\rho+k h+k, 1+\sigma+t k) .
$$

Provided

(i) $\xi, \eta, \varepsilon, v, \sigma, \omega, \mu, \rho, \gamma, \lambda \in \mathbb{C}$;

$\Re(\mu) \geq 0, \Re(\lambda) \geq-1, \Re(\gamma) \geq 0, \Re(\rho) \geq 0, \mathfrak{R}(\eta) \geq 0, \Re(\sigma) \geq 0, \Re(\omega) \geq 0, \Re(v) \geq 0 ; p, q>0$ and $q<\Re(\alpha)+1$

(ii) $\mathfrak{R}(\alpha)>-1$ and $\mathfrak{R}(\beta)>-1$.

$$
\begin{aligned}
I_{4} & =\int_{-1}^{1}(1-x)^{\rho}(1+x)^{\sigma} P_{n}^{(\alpha, \beta)}(x) J_{\eta, \lambda, v, \varepsilon, \omega, p}^{\mu, \xi, \gamma, q}\left[z(1-x)^{h}(1+x)^{-t}\right] d x \\
& =\sum_{k=0}^{\infty} \frac{(\mu)_{\xi k}(\gamma)_{q k}(-z)^{k}}{\Gamma(\eta k+\lambda+1)(v)_{\varepsilon k}(\omega)_{p k}} \int_{-1}^{1}(1-x)^{\rho+h k}(1+x)^{\sigma-t k} P_{n}^{(\alpha, \beta)}(x) d x .
\end{aligned}
$$

Now, by using 12 in 27 , we obtain

$$
\begin{aligned}
& \sum_{k=0}^{\infty} \frac{(\mu)_{\xi k}(\gamma)_{q k}(-z)^{k}}{\Gamma(\eta k+\lambda+1)(v)_{\varepsilon k}(\omega)_{p k}} \frac{(1+\alpha)_{n}}{n !} \\
& =\sum_{k=0}^{\infty} \frac{(-n)_{k}(1+\alpha+\beta+n)_{k}}{(1+\alpha)_{k} 2^{k} k !} \times \int_{-1}^{1}(1-x)^{\rho+k h+k}(1+x)^{\sigma-k t} d x .
\end{aligned}
$$

Using 22 in 28, we get

$$
I_{4}=\frac{2^{\rho+\sigma+1}(1+\alpha)_{n}}{n !} \sum_{k=0}^{\infty} \frac{(-n)_{k}(1+\alpha+\beta+n)_{k}}{(1+\alpha)_{k}(k !)} \times J_{\eta, \lambda, v, \varepsilon, \xi, \omega, p}^{\mu, \xi, q, q}\left[z(2)^{h-t}\right] B(1+\rho+k h+k, 1+\sigma-t k) .
$$

Provided

(i) $\xi, \eta, \varepsilon, v, \sigma, \omega, \mu, \rho, \gamma, \lambda \in \mathbb{C} ; \Re(\mu) \geq 0, \Re(\lambda) \geq-1, \Re(\gamma) \geq 0, \Re(\rho) \geq 0, \Re(\eta) \geq 0, \Re(\sigma) \geq 0, \Re(\omega) \geq 0, \Re(v) \geq$ $0 ; p, q>0$ and $q<\Re(\alpha)+1$. 
(ii) $\mathfrak{R}(\alpha)>-1$ and $\Re(\beta)>-1$.

$$
\begin{aligned}
I_{5} & =\int_{-1}^{1}(1-x)^{\rho}(1+x)^{\sigma} P_{n}^{(\alpha, \beta)}(x) J_{\eta, \lambda, v, \varepsilon, \omega, p}^{\mu, \xi, q, q}\left[z(1+x)^{-h}\right] d x \\
& =\sum_{k=0}^{\infty} \frac{(\mu)_{\xi k}(\gamma)_{q k}(-z)^{k}}{\Gamma(\eta k+\lambda+1)(v)_{\varepsilon k}(\omega)_{p k}} \int_{-1}^{1}(1-x)^{\rho}(1+x)^{\sigma-h k} P_{n}^{(\alpha, \beta)}(x) d x .
\end{aligned}
$$

By using 12 in 30, we get

$$
\sum_{k=0}^{\infty} \frac{(\mu)_{\xi k}(\gamma)_{q k}(-z)^{k}}{\Gamma(\eta k+\lambda+1)(v)_{\varepsilon k}(\omega)_{p k}} \frac{(1+\alpha)_{n}}{n !}=\sum_{k=0}^{\infty} \frac{(-n)_{k}(1+\alpha+\beta+n)_{k}}{(1+\alpha)_{k} 2^{k} k !} \times \int_{-1}^{1}(1-x)^{\rho+k}(1+x)^{\sigma-k h} d x
$$

Again using 22 in 31, we get

$$
I_{5}=\frac{2^{\rho+\sigma+1}(1+\alpha)_{n}}{n !} \sum_{k=0}^{\infty} \frac{(-n)_{k}(1+\alpha+\beta+n)_{k}}{(1+\alpha)_{k}(k !)} \times J_{\eta, \lambda, v, \varepsilon, \omega, p}^{\mu, \xi, \gamma, q}\left[z(2)^{-h}\right] B(1+\rho+k, 1+\sigma-h k) .
$$

Provided

(i) $\xi, \eta, \varepsilon, v, \sigma, \omega, \mu, \rho, \gamma, \lambda \in \mathbb{C}$;

$$
\Re(\mu) \geq 0, \Re(\lambda) \geq-1, \Re(\gamma) \geq 0, \Re(\rho) \geq 0, \Re(\eta) \geq 0, \Re(\sigma) \geq 0, \Re(\omega) \geq 0, \Re(v) \geq 0 ; p, q>0 \text { and } q<\Re(\alpha)+1
$$

(ii) $\mathfrak{R}(\alpha)>-1$ and $\Re(\beta)>-1$.

\section{Special cases}

(i) On setting $\alpha=\beta=\rho=\sigma=0$ and replacing $\delta$ by $\lambda-1$, the integral $I_{2}$ transforms into the following integral involving Legendre polynomials (see [11], [14]):

$$
\begin{aligned}
I_{6} & =\int_{-1}^{1}(1-x)^{\lambda-1} P_{n}(x) P_{m}(x) J_{\eta, \lambda, v, \varepsilon, \omega, p}^{\mu, \xi, \gamma, q}\left[z(1-x)^{h}\right] d x \\
& =2^{\lambda} \sum_{k=0}^{\infty} \frac{(-m)_{k}(-n)_{k}(1+m)_{k}(1+n)_{k}}{(k !)^{2}(k !)^{2}} J_{\eta, \lambda, v, \varepsilon, \omega, p}^{\mu, \xi, \gamma, q}\left[z(2)^{h}\right] B(\lambda+k h+2 k, 1) .
\end{aligned}
$$

(ii) On setting $\alpha=\beta=0$ and replacing $\rho$ by $\rho-1$ and $\sigma$ by $\sigma-1$ then $I_{3}$ transforms into following integral involving Legendre polynomial (see [11], [14]):

$$
\begin{aligned}
I_{7} & =\int_{-1}^{1}(1-x)^{\rho-1}(1+x)^{\sigma-1} P_{n}(x) J_{\eta, \lambda, v, \varepsilon, \omega, p}^{\mu, \xi, \gamma, q}\left[z(1-x)^{h}(1+x)^{t}\right] d x \\
& =2^{\rho+\sigma-1} \sum_{k=0}^{\infty} \frac{(-n)_{k}(1+n)_{k}}{(k !)^{2}} J_{\eta, \lambda, v, \varepsilon, \omega, p}^{\mu, \xi, \gamma, q}\left[z(2)^{h+t}\right] B(\rho+k h+k, \sigma+t k) .
\end{aligned}
$$

(iii) On taking $\alpha=\beta=0$ and replacing $\rho$ by $\rho-1$ and $\sigma$ by $\sigma-1$ then $I_{4}$ transforms into following integral involving Legendre polynomial (see [11], [14]):

$$
\begin{aligned}
I_{8} & =\int_{-1}^{1}(1-x)^{\rho-1}(1+x)^{\sigma-1} P_{n}(x) J_{\eta, \lambda, v, \varepsilon, \omega, p}^{\mu, \xi, \gamma, q}\left[z(1-x)^{h}(1+x)^{-t}\right] d x \\
& =2^{\rho+\sigma-1} \sum_{k=0}^{\infty} \frac{(-n)_{k}(1+n)_{k}}{(k !)^{2}} J_{\eta, \lambda, \nu, \varepsilon, \omega, p}^{\mu, \xi, \gamma, q}\left[z(2)^{h-t}\right] B(\rho+k h+k, \sigma-t k) .
\end{aligned}
$$


(iv) On taking $\alpha=\beta=0$ and replacing $\rho$ by $\rho-1$ and $\sigma$ by $\sigma-1$ then $I_{5}$ transforms into following integral involving Legendre polynomial (see [11], [14]):

$$
\begin{aligned}
I_{9} & =\int_{-1}^{1}(1-x)^{\rho-1}(1+x)^{\sigma-1} P_{n}(x) J_{\eta, \lambda, v, \varepsilon, \omega, p}^{\mu, \xi, \gamma, q}\left[z(1+x)^{-h}\right] d x \\
& =2^{\rho+\sigma-1} \sum_{k=0}^{\infty} \frac{(-n)_{k}(1+n)_{k}}{(k !)^{2}} J_{\eta, \lambda, v, \varepsilon, \omega, p}^{\mu, \xi, \gamma, q}\left[z(2)^{-h}\right] B(\rho+k, \sigma-h k) .
\end{aligned}
$$

(v) On replacing $\varepsilon$ by $\varepsilon-1$ in $I_{1}$ and then by using 5 , we get

$$
\begin{aligned}
& I_{10}=\int_{-1}^{1} x^{\lambda}(1-x)^{\alpha}(1+x)^{\delta} P_{n}^{(\alpha, \beta)}(x) E_{\eta, \varepsilon, v, \sigma, \omega, p}^{\mu, \rho, \gamma, q}\left[z(1+x)^{h}\right] d x \\
& =\frac{(-1)^{n} 2^{\alpha+\delta+1} \Gamma(\delta+k h+1) \Gamma(\alpha+n+1) \Gamma(\delta+k h+\beta+1)}{n ! \Gamma(\delta+k h+\beta+n+1) \Gamma(\delta+k h+\alpha+n+2)} E_{\eta, \varepsilon, v, \sigma, \omega, p}^{\mu, \rho, \gamma, q}\left[z(2)^{h}\right]
\end{aligned}
$$

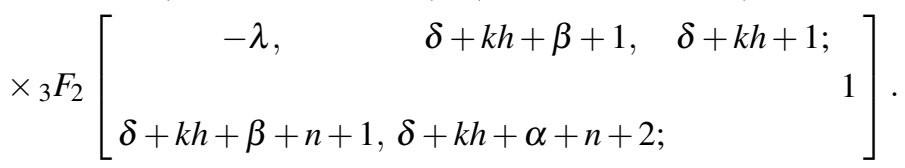

\section{Competing interests}

The authors declare that they have no competing interests.

\section{Authors' contributions}

All authors have contributed to all parts of the article. All authors read and approved the final manuscript.

\section{References}

[1]Ghayasuddin, M, Khan, W. A, A new extension of Bessel-Maitland function and its properties, 70(4)(2018), $292-302$.

[2]Khan, W. A, Nisar, K S, Unified integral operator involving generalized Bessel-Maitland function, Proc. Jangjeon Mathe. Soc. 21(2018), 339 - 346.

[3]Khan, W. A, Nisar, K S, Beta type integral formula associated with Wright generalized Bessel function, Acta Math. Univ. Comenianae, 1(2018), 117-125.

[8]Khan, W. A, Nisar, K S, Choi, J, An integral formula of the Mellin transform type involving the extended Wright Bessel function, Far East J. Math., 102(2017), 2903-2912.

[4]Ali, M, Khan, W. A, Khan, I. A, On certain integral transform involving generalized Bessel-Maitland function and applications, J. Frac. Calcu. and applications, 11(1) (2020), 173-181.

[5]Ali, M, Khan, W. A, Khan, I. A, Study on double integral operator associated with generalized Bessel-Maitland function, Palestine J. Math., 9(1)(2020), 427-435.

[6]Ali, M, Khan, W. A, Khan, I. A, Beta type integral operator involving generalized Bessel-Maitland function, Tamap J. Math. and Stat., (Accepted).

[7]Khan, W. A, Nisar, K S, Ahmad, M, On certain integral transforms involving generalized Bessel-Maitland function, Submitted. [8]Khan, M. A and Ahmed, S, On some properties of the generalized Mittag- Leffler function, Springer Plus 2013,2:337.

[9]Mittag-Leffler, G.M, Sur la nouvelle fonction $E_{\alpha}(x)$, CR Acad. Sci., Paris, 137(1903), 554-558.

[10]Prabhakar, T.R, A singular integral equation with a generalized Mittag-Leffler function in the kernel, Yokohama Math. J., 19(1971), 7-15.

[11]Rainville, E.D, Special functions, The Macmillan Company, New York, 1960. 
[12]Saxena, V.P, The I-function, Anamaya Publisher, New Delhi, 2008.

[13]Shukla, A.K, Prajapati, J.C, On a generalized Mittag-Leffler function and its properties, J. Math. Anal. Appl., 336(2007), $797-811$. [14]Srivastava, H.M and Manocha, H.L, A treatise on generating functions, Ellis Horwood Limited. Co. New York, 1984.

[15]Salim, T.O and Faraj, A.W, A generalization of Mittag-Leffler function and Integral operator associated with the Fractional calculus, Journal of Fractional Calculus and Applications, 3(5)(2012), 1-13

[16]Salim, T.O, Some properties relating to generalized Mittag-Leffler function, Adv. Appl. Math. Anal., 4(1)(2009), 21-30.

[17]Singh, M, Khan, M.A, Khan, A.H, On some properties of a generalization of Bessel-Maitland function, Int. J. Math. Trends and Tech., 14(1)(2014), 46-54.

[18]Watson, G. N, A treatise on the theory of Bessel functions, Cambridge University Press (1995).

[19]Wiman, A, Uber den fundamental satz in der theorie der funcktionen, $E_{\alpha}(x)$, Acta Math., 29(1905), 191-201. 